\title{
Violencia de género, una oportunidad de cuidado en enfermería desde la teoría de acción comunicativa
}

\author{
Rodríguez-Nava Víctor Federico*, Hernández-Castañón Alejandra**, Olvera-Villanueva \\ Georgina $^{* * *}$, Casique- Casique Leticia****
}

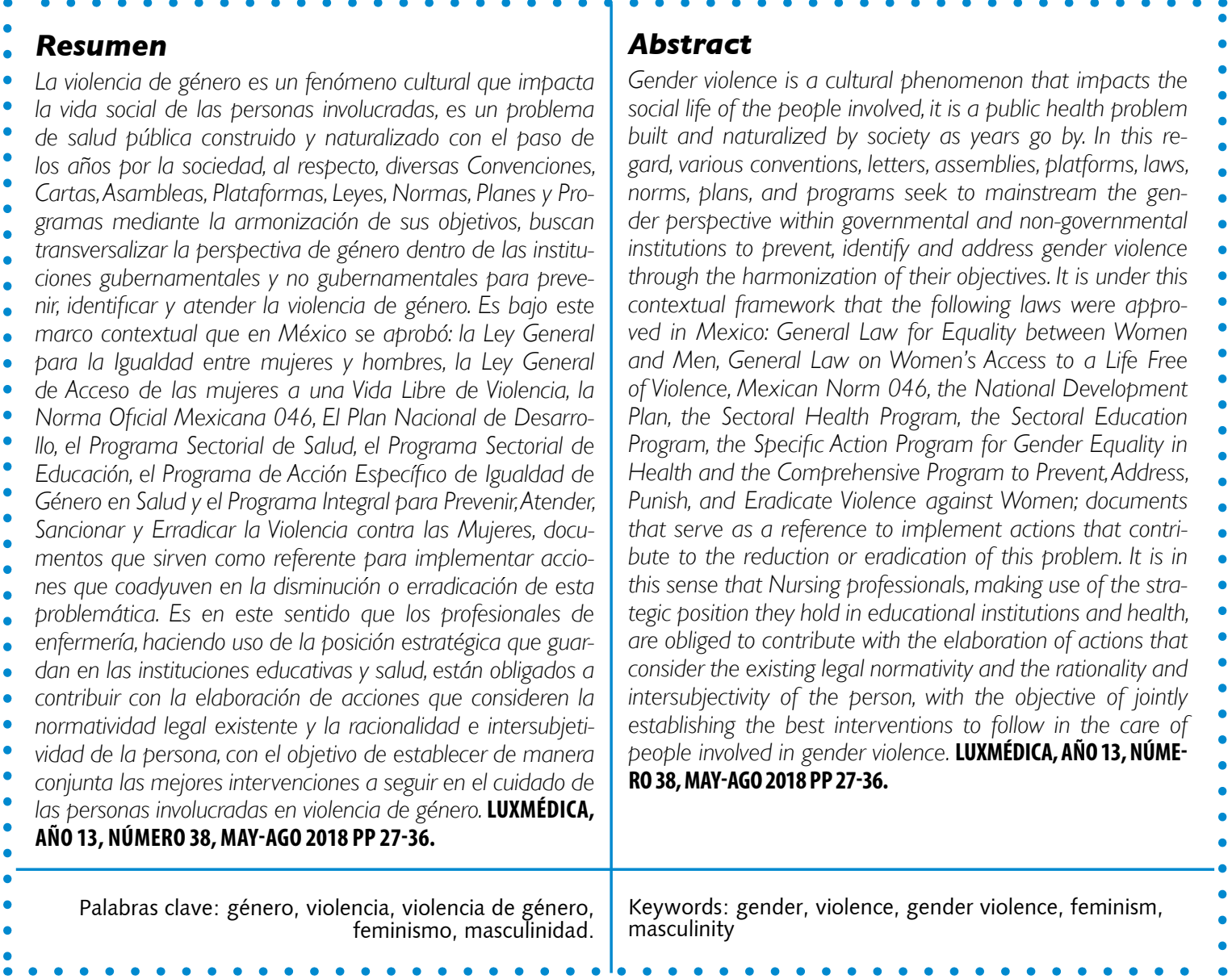

* Estudiante de Doctorado en Ciencias de Enfermería, División de Ciencias de la Salud e Ingenierías, Campus Celaya-Salvatierra, Universidad Autónoma de Guanajuato México.

** Facultad de Ciencias Políticas y Sociales. Universidad Autónoma de Querétaro / Ouerétaro México. (alehdez983@yahoo.commx)

*** División de Ciencias de la Salud e Ingenierías, Departamento de Enfermería y Obstetricia, Campus Celaya-Salvatierra, Universidad de Guanajuato. Guanajuato. México. (olverageorgina@hotmail.com)

**** División de Ciencias de la Salud e Ingenierías, Departamento de Enfermería y Obstetricia, Campus Celaya-Salvatierra, Universidad de Guanajuato. Guanajuato. México. (leticiacc_2004@yahoo.com.mx)

Fecha de recibido: 19 de septiembre 2017

Fecha de aceptación: 15 de enero 2018

Correspondencia: MAE Víctor Federico Rodríguez Nava. Departamento de Enfermería. Edificio 105, Campus Universitario. Universidad Autónoma de Aguascalientes. Avenida Universidad 940 Código postal 20131, Aguascalientes, Ags., México. Teléfono 01 (449) 9108437.

Correo electrónico victorrguez7@hotmail.com 


\section{Introducción}

A lo largo de la historia el ser humano ha ejercido violencia contra sus semejantes como una forma de mostrar dominio y superioridad, por lo que en diversas esferas tanto en el ámbito público y privado se observan escenarios de poder entre sexos que tienen como único objetivo mantener o retomar el control de situaciones que el agresor considera como perdidas ${ }^{1}$, una idea más objetiva sobre la gravedad de este problema lo plantea Ostroski ${ }^{2}$ cuando menciona que basta con hojear las primeras planas de cualquier periódico, en cualquier parte del mundo para identificar la magnitud y ferocidad de este fenómeno, sin olvidar que en ocasiones se puede matar a una persona con la fuerza de la palabra y terminar con alguna vida, sin ser procesado legalmente.

En este orden de ideas, según la Encuesta Nacional Sobre la Dinámica de Relaciones en los Hogares (ENDIREH) $2013^{3}$, la violencia que sufren las mujeres es un fenómeno social, arraigado históricamente y silenciado por décadas, mas ha comenzado a ser visible gracias a defensores de derechos humanos y feministas que se han dado a la tarea de identificarlo y señalarlo tal como sucedió en la Convención para la Eliminación de todas las formas de Discriminación contra la mujer, la Convención Interamericana para prevenir, sancionar y erradicar la violencia contra la mujer de Belém Do Pará y la Conferencia Mundial de Beijing ${ }^{3}$.

Es así que la finalidad del presente artículo es mostrar la responsabilidad que tiene el profesionista de enfermería de establecer acciones que permitan coadyuvar en la disminución o erradicación de la violencia de género mediante la armonización de Leyes, Normas, Planes y Programas aplicables a México sobre esta materia y el referente teórico de la Acción Comunicativa (TAC) de Jürgen Habermas, como método de aproximación entre el profesional de la salud y quien recibirá las acciones establecidas.

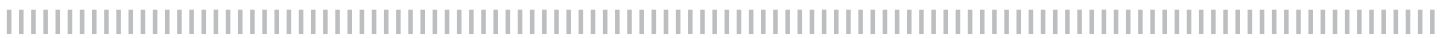

\section{Desarrollo}

\section{Violencia de género y normatividad}

La violencia de género es un problema sociocultural construido históricamente y reconocido por la Organización de las Naciones Unidas (ONU) como el crimen encubierto más frecuente en el mundo ${ }^{4}$, es una epidemia que no respeta fronteras culturales ni de tiempo y una causa importante de años de vida saludables perdidos ${ }^{5}$, situación que repercute en los principales indicadores de morbi-mortalidad a nivel mundial, por lo que afecta los planos individual, familiar, institucional y social de las personas que lo padecen ${ }^{6}$.

Este fenómeno históricamente oculto, cobra nuevamente importancia derivado del proceso de globalización y visibilización a partir de la Convención sobre la Eliminación de todas las formas de Discriminación contra la Mujer (CEDAW), de la cual emanó la Carta Internacional de los Derechos Humanos de las Mujeres (cuyo objetivo es poner fin a la discriminación), misma que fue adoptada por la Asamblea General de 
las Naciones Unidas el 18 de noviembre 1979 y ratificada en México el 23 de marzo de $1981^{7}$.

Es a partir de esta fecha que toma auge el estudio de esta problemática, motivo por el que surgen diferentes documentos a nivel internacional con el objetivo de hacerle frente y erradicarla; entre ellos, los emitidos por la Convención Interamericana para Prevenir, sancionar y erradicar la Violencia contra la Mujer de Belém Do Pará, ratificada por la Asamblea General de la Organización de Estados Americanos (OEA) el 9 de junio de 1994, misma que tiene como propósito "Proteger los derechos humanos de las mujeres" 8 y erradicar las situaciones de violencia de género que puedan afectarlas 9 .

Por su parte, la Plataforma de Acción de Beijing emitida en el año de 1995, promueve los objetivos de igualdad, desarrollo y paz para todas las mujeres del mundo, mediante la eliminación de obstáculos que dificulten la participación activa de la mujer en todas las esferas de la vida pública y privada $^{10}$.

Bajo este contexto, México se suscribe a dichos acuerdos y en el año 2006 publica en el Diario Oficial de la Federación la Ley General para la Igualdad entre mujeres y hombres con el fin de regular y garantizar la igualdad, la no discriminación y equidad entre los sexos ${ }^{9}$; por otra parte en la Ley General de Acceso de las Mujeres a una Vida Libre de Violencia puesta en vigor en el 2007'11, se establecen las bases para la coordinación entre la federación, las entidades federativas y los municipios; de igual forma en los artículos 45 y 46 de esta Ley, se mencionan las competencias de la Secretaría de Educación Pública (SEP) y la Secretaría de Salud (SS) para prevenir, identificar y atender esta problemática. ${ }^{12-13}$

Cabe resaltar que en el Diario Oficial de la Federación se publicó la Norma Oficial Mexicana-190-SSA1-1999, Prestación de servicios de salud. Criterios para la aten- ción médica de la violencia familiar, la cual modificó su nomenclatura a NOM-046SSA2-2005 Violencia familiar, sexual, y contra las mujeres. Criterios para la prevención y atención ${ }^{14}$. Sin embargo, no ha sido empleada de manera general por los profesionistas del área de la salud, debido a que aún en nuestros días, no reconocen la violencia como un problema de salud ${ }^{15}$, ni lo establecido en la normatividad para las instituciones del Sistema Nacional de Salud en carácter de obligatoriedad.

Con referencia a lo anterior, el Plan Nacional de Desarrollo 2013-2018 establece como objetivo: "Llevar a México a su máximo potencial" a través de cinco metas nacionales, a saber: I) México en paz, II) México incluyente, III) México con educación de calidad, IV) México próspero y V) México con responsabilidad global, todas ellas transversalizadas por tres estrategias I) Democratizar la productividad, II) Gobierno cercano y moderno y III) Perspectiva de género $^{16}$, es específicamente en esta última estrategia que el gobierno federal busca fomentar un proceso de cambio dentro de las instituciones públicas y privadas, con el objetivo de evitar que se reproduzcan roles y estereotipos de género, lo que sin lugar a dudas, contribuye de manera directa a cumplir los compromisos asumidos por México al ratificar la Convención sobre la Eliminación de todas las Formas de Discriminación contra la Mujer. ${ }^{16}$

Según el Instituto Nacional de las Mujeres (INMUJERES), la perspectiva de género busca mostrar que las diferencias entre mujeres y hombres se dan no solo por su determinación biológica, sino también por las diferencias culturales asignadas a los seres humanos, por lo que cuestiona las desigualdades en el trato, acceso y oportunidades de las personas ${ }^{17}$, es por ello que en el Plan Nacional de Desarrollo, se establecen las acciones a considerar por todas las instituciones de gobierno, con el objetivo de garantizar el respeto y protección 
de los derechos humanos, la eliminación de la discriminación y el establecimiento de medidas especiales orientadas a erradicar la violencia de género. ${ }^{16}$

Ante esta situación, además de la ya citada Norma Oficial Mexicana 046, se elabora el Programa de acción específico de igualdad de género en salud ${ }^{18}$ y el Programa Integral para Prevenir, Atender, Sancionar y Erradicar la Violencia contra las Mujeres ${ }^{19}$, dentro de los cuales se establecen acciones encaminadas a evitar la discriminación y violencia dirigida hacia las mujeres.

Por lo anterior y de acuerdo con los razonamientos que se han venido realizando, la propuesta de Transversalización de Género dentro del ámbito educativo y de salud, tienen una base teórica a partir de la cual es posible establecer acciones para erradicar la violencia, modificar roles sociales y con ello coadyuvar a la igualdad entre hombres y mujeres.

Por lo que este contexto, es idóneo para que el personal de Enfermería asuma un papel protagónico en el cuidado de la persona a partir de la promoción de una cultura de igualdad y no violencia entre hombres y mujeres, incorporando en sus programas de estudios acciones con una perspectiva de género y énfasis en la prevención de la violencia, es decir, que en la formación de Enfermería se desarrollen acciones que permita proporcionar cuidados profesionales a las personas involucradas, además de que se elaboren programas de capacitación para el personal del sector salud relacionados con esta temática, lo que permitirá proponer nuevos modelos de cuidado dirigidos a la población, tal como se menciona en la Ley General de Acceso de las Mujeres a una Vida Libre de Violencia ${ }^{11}$ y de esta forma abonar en el cumplimento de la normatividad internacional y nacional vigente en torno a la paz, justicia e igualdad de género. ${ }^{20-21-22}$

Por lo anterior, es válido considerar que en la Atención Primaria a la Salud, el cui- dado profesional e integral en torno a la violencia de género, es una obligación del profesionista de Enfermería.

\section{El cuidado profesional de enfermería ante Ia violencia de género}

Las instituciones de salud están concebidas bajo un modelo medicalizado enfocado más al proceso curativo que preventivo ${ }^{16}$, razón por la cual es necesario que el profesional de Enfermería se sensibilice ante esta situación y considerando el caso particular de la violencia de género, implemente planes de acción que cuenten con el respaldo legal, científico y de empoderamiento de la persona, planes de acción que permitan transformar la idea de que la violencia es "Un problema natural y de carácter privado" y sea observado el hecho como "Un problema de salud social" que debe ser prevenido, identificado y atendido en las unidades de salud ${ }^{13}$

Si bien es cierto que se cuenta con la Norma Oficial Mexicana (NOM 046), en general el personal de salud no se ha involucrado en su seguimiento y por tanto no se tiene una sensibilización ante esta problemática, razón por la que Enfermería, no cuenta con un plan de cuidados consensuado para la prevención, identificación y atención de las víctimas de violencia de género ${ }^{23}$, es necesario recordar que unas de las características del proceso de Enfermería, es valorar al paciente como un ser individual y único en sus dimensiones físicas, sociales, culturales, espirituales y afectivas $^{24}$, situación por lo cual se considera que Enfermería tiene la oportunidad de brindar cuidado profesional a cada persona, adaptando ésta a su contexto sociocultural, sus necesidades específicas y los factores globales que influyen en él, tales como la visión del mundo de la vida, la estructura social, el lenguaje, la etnohistoria y el contexto ambiental de cada persona en particular. ${ }^{25}$ 
Como resultado de lo anterior, el profesional de Enfermería tiene una posición estratégica dentro de la sociedad, (Entendida por Habermas como el mundo de la vida) ${ }^{26}$, debido a que son los profesionales del área de la salud con mayor cantidad de plazas, el primer contacto con la persona que requiere un cuidado especifico, por lo que según Linares ${ }^{23}$, los pacientes tienden a percibir al profesional de Enfermería como más humanos, cercanos y accesibles en comparación a otros profesionales del área, por lo que Enfermería es capaz de identificar algunas características definitorias que oriente la elaboración de diagnósticos de Enfermería tales como: riesgo de suicidio, desesperanza, riesgo de lesión, deterioro de la integridad cutánea, síndrome postraumático, procesos familiares disfuncionales, trastorno del patrón del sueño, temor, síndrome del trauma pos-violación, baja autoestima, ansiedad, afrontamiento ineficaz, aislamiento social, conflicto de decisiones, tendencia a adoptar conductas de riesgo para la salud, insomnio, entre algunos otros que den indicio de la existencia de violencia de género ${ }^{23,27}$.

En este orden de ideas, las intervenciones preventivas de Enfermería se pueden implementar según Robert Gordon ${ }^{28}$ de tres formas: a) De prevención universal, en donde se dan acciones dirigidas a la población en general, b) De prevención selectiva o focalizada, con acciones dirigidas a personas que integran sub grupos de la población y que por sus características sean susceptibles y estén expuestas a un mayor riesgo y c) De prevención indicada, que son medidas dirigidas a personas que manifiesten un factor de riesgo, condición o signo de estar ya dentro de la problemática.

Aunado a lo anterior, y con el objetivo de identificar y atender esta problemática, la Ley General para la Igualdad entre Mujeres y Hombres, la Ley General de Acceso a una Vida Libre de Violencia, la Norma Oficial Mexicana 046, El Plan Nacional de Desarrollo, El Programa Sectorial de Salud, el Programa Sectorial de Educación, el Programa de Acción Especifico de Igualdad de Género en Salud y el Programa Integral para Prevenir, Atender, Sancionar y Erradicar la Violencia contra las Mujeres, entre otros ya citados con anterioridad, son el referente para establecer acciones de Enfermería para prevenir identificar y atender la violencia de género tal como se ve en los cuadros. ${ }^{1-5}$

\section{Cuadro I}

\section{Acciones de Enfermería universales para prevenir la violencia de género}

- Gestionar campañas permanentes de difusión para prevenir la violencia de género.

- Fomentar los derechos humanos y las relaciones de equidad e igualdad en el ámbito educativo, salud y familiar.

- Hablar sobre las causas y consecuencias de la violencia de género.

- Difundir el contenido y aplicación de la Ley General de Acceso de las Mujeres a una Vida Libre de Violencia y la Norma Oficial Mexicana 046 entre las y los usuarios de los servicios de salud, estudiantes, trabajadores y aquellos privados de su libertad.

- Sugerir la eliminación dentro de las instituciones de salud y educativas contenido sexista y discriminatorio.

- Incorporar en planes y programas de estudios de enfermería la perspectiva de género, para fomentar la igualdad entre mujeres y hombres.

- Establecer campañas que promuevan el derecho a una vida sin violencia y la resolución pacífica de los conflictos.

- Promover la sensibilización a profesionales de la comunicación sobre causas e impactos de la violencia.

- Promover relaciones familiares igualitarias y respetuosas a través de los medios de comunicación.

- Impartición de programas de capacitación en tópicos relacionados con la violencia de género, dirigidos tanto a hombres como mujeres.

Fuente:10-14, 16, 18, 19, 29, 30 
Violencia de género, una oportunidad de cuidado en enfermería desde la teoría

\section{Cuadro 2}

\section{Acciones de Enfermería selectivas para prevenir la violencia de género}

\section{Selectivas:}

- Capacitar y sensibilizar a funcionarios, autoridades, servidores públicos, personal docente y del área de la salud en: Perspectiva de género, derechos humanos y la no violencia.

- Promover estilos de vida saludables que incluyan el desarrollo de las responsabilidades compartidas al interior de las familias.

Fuente:10-14, 16, 18, 19, 29, 30

\section{Cuadro 3}

\section{Acciones de Enfermería indicadas para prevenir la violencia de género}

- Impartir consejería en el servicio médico, educativo y laboral con personal capacitado, sobre las alternativas para la prevención, identificación y atención en torno a la violencia de género.

- Fortalecer la vinculación entre la sociedad, el ámbito escolar, de salud y laboral para acotar la violencia mediante acciones integrales.

- Integrar una Red de Promotores de no violencia y nuevas masculinidades en la comunidad, espacios educativos y el área de salud.

- Realizar tamizaje de manera rutinaria y en casos probables.

Fuente:10-14, 16, 18, 19, 29, 30

\section{Cuadro 4}

\section{Acciones de Enfermería para identificar la violencia de género}

- Sensibilizar al personal que participa en la identificación y atención en violencia de género.

- Establecer en el sistema de salud, escolar y laboral mecanismos de detección de la violencia de género.

- Crear áreas especializadas para detectar y denunciar la violencia en los centros de trabajo, las unidades de salud y el área escolar, facilitando la accesibilidad al área.

- Promover la aplicación de procedimientos de detección oportuna de violencia de género mediante marcadores de riesgo confiables.

- Registrar la valoración de Enfermería de la o del usuario afectado por violencia de género en el expediente clínico en forma detallada, clara y precisa, colocando el grado de riesgo.

- Elaborar un test corto con alta sensibilidad y especificidad para la detección de posibles personas involucradas en la violencia de género.

Fuente:10-14, 16, 18, 19, 29, 30 


\section{Cuadro 5}

\section{Acciones de Enfermería para atender la violencia de género}

- Realizar investigaciones interdisciplinarias e interinstitucionales, que permitan mejorar la calidad de la prevención, identificación, atención y rehabilitación de la violencia de género para estar en condiciones de afrontarlo mejor.

- Sensibilizar al personal que participa en el cuidado de personas involucradas en violencia de género para que proporcionen cuidados sin discriminación o misoginia.

- Capacitar en derechos humanos, género, protección integral, derechos de la infancia, interculturalidad y trámites de referencia a instancias especializadas a las personas que participan en el cuidado de las víctimas.

- Implementar y evaluar los programas relacionados con la erradicación de la violencia de género.

- Promover políticas institucionales para erradicar la violencia de género que ocurre en los servicios de salud.

- Implementar programas y servicios reeducativos para víctimas y agresores.

- Integrar un registro nacional de feminicidios, homicidios dolosos y por razones de género con datos sociodemográficos de víctimas y agresores.

- Elaborar modelos de prevención, identificación y atención con equidad y género, dirigidos a generadores y víctimas de violencia.

- Referir a las y los usuarios involucrados en situación de violencia de género a servicios de atención especializada.

- Seguimiento a las personas involucradas en violencia de género.

Fuente: $10-14,16,18,19,29,30$

Es así que, considerando los documentos antes mencionados, a partir de Enfermería es posible establecer acciones de prevención, identificación y atención de bajo costo, con sustento legal, que favorezcan la transversalidad de género dentro de las instituciones educativas y de salud ${ }^{10}$.

La teoría de la acción comunicativa como referente teórico para el cuidado enfermero en la prevención, identificación y atención de las personas involucradas en violencia de género

Se puede reconocer que para erradicar la violencia de género es indispensable el trabajo con hombres desde la reeducación, persona que en palabras de Habermas se convierte en el alter ego del interlocutor ${ }^{31}$ y debe ser considerado en toda acción tendiente a prevenir, identificar y atender a personas involucradas en violencia de género.

Desde esta perspectiva, la teoría de Jürgen Habermas (Filósofo y sociólogo Alemán y uno de los intelectuales más influyentes de la llamada "Segunda Generación" de la escuela de Frankfurt en Alemania) ${ }^{26}$, propone el análisis de la sociedad a partir de su Teoría de la Acción Comunica- tiva $(T A C)^{31}$, en la que presenta dos formas de racionalidad a saber: a) La racionalidad sustantiva del mundo de la vida, considerada como un lugar en el que el hablante y oyente salen al encuentro e interactúan con el objetivo de lograr acuerdos sobre algún tema en particular y b) La racionalidad formal del sistema, la cual es el punto de vista de los sujetos que actúan en la sociedad de alguien no implicado ${ }^{32-33}$, es así que, considerando las racionalidades anteriormente descritas, es posible plantear la necesidad de interacción de la persona y las instituciones de salud desde sus respectivas racionalidades, con el único fin de proporcionar cuidados de Enfermería que permitan prevenir, identificar y atender personas involucradas en violencia de género de manera consensuada.

Es necesario mencionar que a través de la TAC, Habermas critica al unilitarismo, cuestiona el tecnicismo y propone percibir al hombre y su mundo a través de una ética en la que se destruyan las barreras de comunicación entre los interlocutores ${ }^{34}$, situación que como consecuencia, favorece al menos cuatro situaciones para la práctica del profesional de Enfermería al 
interactuar con la persona en el mundo de la vida: a) Permite el establecimiento de relaciones interpersonales basadas en el diálogo, b) Admite la elaboración de planes de acción considerando argumentos, normas o reglas de la persona, c) Favorece el consenso, entendimiento, comprensión, inteligibilidad e intersubjetividad entre los participantes, evitando en todo momento la colonización del mundo de la vida de la persona afectada y d) Concede la validación de afirmación mediante los actos del habla $26,31,35$.

Como consecuencia de lo anterior y refiriendo a Villarreal-Reyna ${ }^{36}$, en su artículo titulado La Teoría Crítica y su Contribución al Desarrollo del Conocimiento en Enfermería, se rescata la idea de que la aplicabilidad de la TAC en la práctica de Enfermería es una opción para que el diálogo entre el profesional de Enfermería y la persona involucrada en el problema de violencia de género, promueva una elección racional del plan de acción a seguir por los interlocutores a partir del consenso, dando pauta con ello a la emancipación de la persona, en tanto que Duque-Páramo ${ }^{35}$ menciona que se deben desarrollar planes de acción y proyectos participativos diseñados de manera conjunta con las personas con quienes se trabaja en los hospitales, clínicas y ámbitos comunitarios, espacios en los que Enfermería tiene injerencia y por ende la posibilidad de proporcionar los cuidados profesionales a víctimas relacionadas con la problemática de violencia de género.

Es así que, en el marco de los documentos oficiales antes mencionados, se puede afirmar que en la prevención, identificación y atención de la violencia de género, Enfermería puede contribuir con la elaboración de acciones efectivas que consideren a la persona y sus respectivas racionalidades mediante una coordinación comunicativa y respetuosa de las acciones a realizar ${ }^{35}$, tomando en cuenta los beneficios que ofrece la TAC para la profesión de enfermería, al considerar a la persona como un ser que adquiere experiencia, que dialoga y construye cuidado en la intersubjetividad ${ }^{31-32}$ y no mediante la colonización del mundo de la vida es decir, al sólo repetir modelos hegemónicos ${ }^{37}$.

En este contexto, el cuidado de enfermería establecido de manera conjunta con la persona y basado en el diálogo, contribuye al establecimiento del mejor plan de cuidados que permita prevenir, identificar, atender a las personas víctimas de violencia de género y con ello coadyuvar en la erradicación de este problema, dado que la coparticipación en el cuidado de la persona, de las instituciones educativas y de salud, permite a los interlocutores seleccionar y ejecutar planes de acción consensuados y en común acuerdo 35,38

\section{Conclusiones}

A pesar de que se cuenta con diferentes normatividades en los planos internacional y nacional, éstas no han alcanzado los resultados esperados entorno a la disminución de la violencia de género, por lo que se plantea la implementación de acciones tanto en los ámbitos educativos como de salud, con el objetivo de participar en el cuidado a víctimas inmersas en este problema, considerando en todo momento la normatividad legal existente y la TAC para establecer de manera conjunta el mejor plan de acción a seguir. 


\section{Bibliografía}

1. Varela N, Freire E. Feminismo para principiantes. 1a ed. Barcelona: Ediciones B; 2005.

2. Ostrosky S, Feggy. Mentes asesinas. 1a ed. México, D.F: Hachette Filipacchi Expansio?n; 2008.

3. Instituto Nacional De Estadística Y Geografía. Panorama de violencia contra las mujeres en los Estados Unidos Mexicanos. ENDIRE [Internet]. 2013; 1(1):1-101. [consulta 15 Junio 2017]. Disponible en: http://internet.contenidos.inegi.org.mx/contenidos/productos/prod_serv/contenidos/espanol/ bvinegi/productos/estudios/sociodemografico/mujeresrural/2011/702825048327.pdf

4. Ruíz Pérez I. Violencia contra la mujer y salud. [Internet]. 1-20. [consulta 17 Junio 2017]. Disponible en: http://www.msssi.gob.es/organizacion/sns/planCalidadSNS/pdf/equidad/04modulo_03.pdf

5. Secretaria de Salud. Informe Nacional Sobre Violencia y Salud. [Internet], 2006; 1-542. [consulta 19 Julio 2017]. Disponible en: http://www.salud.gob. $\mathrm{mx} /$ unidades/cdi/documentos/InformeNalsobreViolenciaySalud.pdf

6. Herrero de Frutos MT. Plan de cuidados en mujer inmigrante víctima de violencia de género. [Internet]. Enfermería, Fisioterapia y Podología. 2014; 6(2):151. [consulta 16 Junio 2017]. Disponible en: http:// www.revistareduca.es/index.php/reduca-enfermeria/article/viewFile/1701/1720

7. Organización de las Naciones Unidas. Convención Sobre La Eliminación De Todas Las Formas De Discriminación Contra La Mujer [Internet].1981. 1-9. [consulta 14 Junio 2017]. Disponible en: http:// cedoc.inmujeres.gob.mx/documentos_download/100039.pdf

8. Organización De Las Naciones Unidas. Informe de la Cuarta Conferencia Mundial sobre la Mujer. [Internet]. 1996. 1-238. [consulta 2 Junio 2017]. Disponible en: http://www.un.org/womenwatch/daw/ beijing/pdf/Beijing\% 20full\% 20report\% 20S.pdf

9. Cámara de Diputados del H. Congreso de la Unión. Ley General Para La Igualdad Entre Mujeres Y Hombres [Internet]. 2016. [consulta 3 Junio 2017]. Disponible en: http://www.diputados.gob.mx/LeyesBiblio/pdf/LGIMH_240316.pdf

10. Organización de las Naciones Unidas Mujeres. Declaración y Plataforma de Acción de Beijing. [Internet]. 2014. 1-316. [consulta 21 de junio de 2017]. Disponible en: http://beijing20.unwomen.org/ / media/headquarters/attachments/sections/csw/ bpa s final web.pdf

11. Cámara de Diputados del H. Congreso de la Unión. Ley General De Acceso De Las Mujeres A Una Vida Libre De Violencia [Internet]. 2007. 1-37. [consulta 10 Mayo 2017]. Disponible en: http://www.diputados.gob.mx/LeyesBiblio/pdf/LGAMVLV_220617. pdf

12. Secretaria De Educación Púbica. Programa Sectorial de Educación 2013-2018 [Internet]. 2013. 1-117. [consulta 18 Mayo 2017]. Disponible en: http:// www.sep.gob.mx/work/models/sep1/Resource/4479/4/images/programa_sectorial_de_educa- cion 20132018 web.pdf

13. Secretaria De Salud. Programa Sectorial De Salud 2013-2018 [Internet]. 2013. 1-138. [consulta 19 Junio 2017]. Disponible en: http://www.conadic. salud.gob.mx/pdfs/sectorial_salud.pdf

14. Secretaria De Salud. Norma Öficial Mexicana (NOM046-SSA2-2005). Violencia familiar, sexual y contra las mujeres. Criterios para la prevención y atención [Internet]. 2009. 1-34. [consulta 19 Junio 2017]. Disponible en: http://www.inm.gob.mx/static/ Autorizacion_Protocolos/SSA/Violencia_familiar_ sexual_y_contra_las_mujeres_criterios_par.pdf

15. Coll Vinent B, Echeverría T, Farràs Ú, et al. El personal sanitario no percibe la violencia doméstica como un problema de salud. Gac Sanit. 2008; 22(1):7-10. [consulta 19 Junio 2017]. Disponible en: http://scielo.isciii.es/scielo.php?script=sci_pdf\&pid = S0213$91112008000100002 \& \operatorname{lng}=e s \& n r m=i s o \& t \operatorname{lng}=e s$

16. Gobierno De La Republica. Plan Nacional De Desarrollo 2013-2018. [Internet]. 2013. 1-184. [consulta 28 Mayo 2017]. Disponible en: http://pnd.gob.mx/ wp-content/uploads/2013/05/PND.pdf

17. Instituto Nacional De Las Mujeres. Glosario de género [Internet]. 2008. 1-193 [consulta 25 Mayo 2017]. Disponible en: http://www.aguascalientes.gob.mx/ iam/LIBROS\% 20PDF's/GLOSARIO\% 20DE\% 20 G\% C3\% 89NERO\% 20ELECTRONICO.pdf

18. Programa Sectorial De Salud. Igualdad de Género en Salud [Internet]. 2013. 1-84. [consulta 21 Julio 2017]. Disponible en: http://www.gob.mx/cms/ uploads/attachment/file/9981/IgualdaddeGeneroenSalud.pdf

19. Gobierno De La Republica. Programa Integral Para Prevenir, Atender, Sancionar Y Erradicar La Violencia Contra Las Mujeres [Internet]. 2014. 1-80. [consulta 6 Junio 2017]. Disponible en: http://conavim.gob. $\mathrm{mx} /$ work/models/CONAVIM/Resource/114/2/ images/PIPASEVM_DOF_2014-2018.pdf

20. User S. Los Objetivos de Desarrollo Sostenible y la Igualdad de Género [Internet]. Equidad.org.mx. 2015. [consulta 19 Junio 2017]. Disponible en: http://www.equidad.org.mx/index.php/es/201410-20-22-31-06/388-los-objetivos-de-desarrollosostenible-y-la-igualdad-de- género

21. Programa de las Naciones Unidas Para el Desarrollo en México. Objetivo 5: Igualdad de género [Internet]. 2015. [consulta 9 Junio 2017]. Disponible en: http://www.mx.undp.org/content/mexico/es/ home/post-2015/sdg-overview/goal-5.html

22. Programa de las Naciones Unidas para el Desarrollo en México. Objetivo 16: Paz, justicia e instituciones fuertes [Internet]. 2015. [consulta 8 Junio 2017]. Disponible en: http://www.undp.org/content/ mexico/es/home/post-2015/sdg-overview/goal-16. html

23. Linares Cano J. Análisis e intervención de Enfermería en la Violencia de Género. Importancia del diagnóstico dentro del Equipo de salud. III Congreso para el Estudio de la Violencia contra las Mujeres: Justicia y Seguridad. Nuevos retos [Internet]. Congresoestudioviolencia.com. 2012. [consulta 12 Junio 2017]. Disponible en: http://www. 
violenciageneroasistenciavictimas.es/index.php/ lineas-de-actuacion/congreso-vg/iii-congreso/ articulos-cientifico-tecnicos/136-analisis-e-intervencion-de-enfermeria-en-la-violencia-de-generoimportancia-del-diagnostico-dentro-del-equipo-desalud

24. Rodríguez Jiménez $S$, Cárdenas Jiménez $M$, Pacheco Arce $A L$, et al. Una mirada fenomenológica del cuidado de Enfermería. Enferm Univ. [Internet]. 2014; 11(4):145-53. [consulta 19 Junio 2017]. Disponible en: http://www.elsevier.es/es-revista-enfermeriauniversitaria-400-articulo-una-mirada-fenomenologica-del-cuidado-S1665706314709273

25. Tomey A, Alligood M. Modelos y teorías en Enfermería. 8a ed. Madrid, España: Elsevier; 2014.

26. Garrido Vergara L. Habermas Y La Teoría De La Acción Comunicativa [Internet]. Razón Y Palabra. 2011; (75): 1-19. [consulta 23 Junio 2017]. Disponible en: http://www.razonypalabra.org.mx/N/N75/ ultimas/38_Garrido_M75.pdf

27. Heather Herdman T, Shigemi K. Diagnósticos Enfermeros, Definiciones y Clasificación 2015-2017. 10a ed. Edición Hispanoamericana; Chichester, UK.: Wiley-Blackwell; 2016.

28. Fernández Hermida JR, Secades Villa R. Ética y prevención del consumo de drogas. [Internet]. Revista Española de Drogodependencia. 2010; 35(1):4-20. [consulta 6 Junio 2017]. Disponible en: http://www. aesed.com/descargas/revistas/v35n1 1.pdf

29. Cámara de Diputados del H. Congreso de la Unión. Ley De Planeación. [Internet]. 2016. [consulta 29 Mayo 2017]. Disponible en: http://www.snieg.mx/ contenidos/espanol/normatividad/marcojuridico/leydeplaneacion.pdf

30. Gobierno De La Republica. Programa Para La Igualdad Entre Mujeres Y Hombres [Internet]. 2014. 1-32. [consulta 15 Junio 2017]. Disponible en: http:// www.ceav.gob.mx/wp-content/uploads/2012/06/ Programa-para-la-Igualdad-entre-Mujeres-y-Hombres-2014-2018-final.pdf
31. Habermas J, Jiménez Redondo M. Teoría de la acción comunicativa. Madrid: Ca?tedra; 1989.

32. Díaz Montiel ZC, Márquez Fernández, Alvarado B. La Modernidad en Habermas: Del "sistema" (represor) al "mundo de Vida" (liberador). Revista de Artes y Humanidades UNICA [Internet]. 2008; 9(21):7197. [consulta 11 Junio 2017]. Disponible en: http:// www.redalyc.org/pdf/1701/170118398004.pdf

33. Reyes Cueto A. Crisis De La Racionalidad En El Mundo Moderno [Internet]. Bibliotecadigital.academia.cl. 2010. 1-85. [consulta 12 Junio 2017]. Disponible en: http://bibliotecadigital.academia.cl/bitstream/handle/123456789/87/TSOC131. pdf; jsessionid=AC4B6 OA34398835D2387075E455FEF69? sequence $=1$

34. Macedo Costa K, De Almeida Rebouças C, Freitas da Silva GR, et al. Enfermero-Docente: Aspectos Interrelacionados Con Su Formación. Enfermería Global [Internet]. 2010; 19(1):1-15. [consulta 16 Junio 2017]. Disponible en: http://scielo.isciii.es/pdf/eg/ n19/reflexion2.pdf

35. Duque Páramo MC. Solidaridad y ciencia. Reflexiones en el contexto del cuidado de Enfermería como interacción comunicativa. Investigación en Enfermería: Imagen y Desarrollo [Internet]. 2011; 13(2):101112. [consulta 19 Junio 2017]. Disponible en: http:// www.redalyc.org/articulo.oa?id $=145222559007$

36. Villarreal Reyna M, Salazar González B. La Teoría Crítica y su Contribución al Desarrollo del Conocimiento en Enfermería. Desarrollo Científ Enferm [Internet]. 2006; 14(9):339-341. [consulta 27 Junio 2017]. Disponible en: http://www.index-f.com/ dce/14pdf/14-339.pdf

37. Rich Ruiz $M$, Martins $M$, Rodríguez Borrego $M$. Tecnología Y Comunicación En El Cuidado Hospitalario A Enfermos Crónicos Desde La Perspectiva De Habermas. Texto Contexto Enferm [Internet]. 2014; 23(3):704-711. [consulta 29 Mayo 2017]. Disponible en: http://dx.doi.org/10.1590/010407072014003390013

38. Habermas J. Teoría de la acción comunicativa. Madrid: Taurus; 1987. 